\title{
Using Runtime Measurements and Historical Traces for Acquiring Knowledge in Parallel Applications
}

\author{
Luciano José Senger ${ }^{1}$, Marcos José Santana ${ }^{2}$, and Regina Helena Carlucci Santana ${ }^{2}$ \\ 1 Universidade Estadual de Ponta Grossa, Departamento de Informática \\ Av. Carlos Cavalcanti, 4748 Zip Code 84030-900 Ponta Grossa, PR, Brazil \\ 2 Universidade de São Paulo, Instituto de Ciências Matemáticas e de Computação \\ Av. Trabalhador Saocarlense, 400 PO Box 668 Zip Code 13560-970 São Carlos, SP, Brazil \\ \{1jsenger, mjs, rcs\}@icmc.usp.br
}

\begin{abstract}
A new approach for acquiring knowledge of parallel applications regarding resource usage and for searching similarity on workload traces is presented. The main goal is to improve decision making in distributed system software scheduling, towards a better usage of system resources. Resource usage patterns are defined through runtime measurements and a self-organizing neural network architecture, yielding an useful model for classifying parallel applications. By means of an instance-based algorithm, it is produced another model which searches for similarity in workload traces aiming at making predictions about some attribute of a new submitted parallel application, such as run time or memory usage. These models allow effortless knowledge updating at the occurrence of new information. The paper describes these models as well as the results obtained applying these models to acquiring knowledge in both synthetic and real applications traces.
\end{abstract}

\section{Introduction}

One of the challenges in parallel and distributed is to develop scheduling algorithms that assigns the tasks of parallel applications to the heterogeneous machines. Many researchers have demonstrated that using parallel application knowledge may improve the scheduling decisions on multiprogrammed multiprocessor systems [1][2]. Nevertheless, most of the work has assumed that such knowledge is available a priori and does not provide effective indications to obtain it. There are commonly three main sources to obtain knowledge: the description of applications requirements provided by the user (or programmer) who submits the parallel application to the system; historical traces of all applications executed in a specific system over a time period, and runtime measurements from parallel applications. Among these knowledge sources, historical traces and runtime measurements have demonstrated a great potential to provide information aiming at classifying parallel applications and obtaining knowledge [3][4].

This paper presents two models for knowledge acquisition in parallel applications aiming at improving software scheduling decisions. The first model aims for parallel applications classifying, regarding behavior on resource usage. Compared to previous work, these models have two novel aspects. First, they allow updating of acquired knowledge at the occurrence of new information. Second, they can be used to improve different scheduling policies, not aimed at particular scheduling strategies. 


\section{Local Knowledge Acquisition}

The main goal of the model described in this section is the knowledge acquisition from the execution of tasks which compose parallel applications, classifying parallel applications regarding its behavior on resource utilization by classes such as CPU Bound, I/O Bound and Communication Intensive [5]. This model allows the utilization of knowledge acquired in previous runs of the applications and the knowledge updating in presence of new information. The model aiming at identifying the resource usage phases during the parallel application execution using runtime measurements. Collected observations are separated by intervals of time $T_{o}$. A sample is created for each $N_{o}$ observations, characterizing an Euclidean vector with dimension $d$. These samples samples are grouped in clusters. An important aspect of the model is how to carry out the clustering of the vectors. Since an on-line strategy is desired, it is adopted the utilization of the ART 2A self-organizing neural architecture [6] as the solution. The ART 2A neural network allows incremental clustering and classification of a pattern set composed of continuous values [7]. A significance matrix $S M$ [8], which is composed by a set of significance values $S V_{i j}$, is employed to assign a label for each cluster. The significance values are obtained directly from the ART 2A long term memory.

The model was evaluated using an input feature vector with dimension $d=7$ : CPU user time; memory usage; read and write file operations and send and receive network operations. The model was tested on the PSTSWM (Parallel Spectral Transform Shallow Water Model) [9] application. The PSTSWM is a representative compact application that solves the nonlinear shallow water equations on a rotating sphere using the spectral transform method. The model parameters were $T_{o}=1000 \mathrm{~ms}$ and $N_{o}=1$ and the results obtained are illustrated in Table 1 The majority of application phases (about 56\%) were classified as communication intensive (cluster $k=1$ ). The other phases were classified as communication intensive ( $k=2$ and $k=4$ ) and CPU-Bound (cluster $k=3$ ).

\section{Global Knowledge Acquisition}

The local knowledge acquisition model presented in the previous section aiming at knowledge acquiring of a particular application resource usage. Scheduling algorithms often need to know some application attribute in advance of its execution (e.g. run time, memory usage and сри time). The workload traces can be treated as a database of previous experiences (experience base) about parallel applications and depending on the scheduling algorithm requirements, some attribute, like run time or memory usage, can be considered as the attribute to be predicted. The global knowledge acquisition model described in this section is constructed using instance-based learning. Instance-based learning (IBL) is an approach which find similar instances in an experience base aiming at approximating real-valued or discrete-valued target functions [10]. IBL algorithms compute the similarity between a new query instance and the experience base instances, returning a set of related instances as output.

The output attribute considered to predict is the parallel application run time, although any attribute can be used as output in our IBL algorithm implementation. Many authors have demonstrated that parallel applications run time knowledge can be very useful 
to space-sharing scheduling algorithms [11]. Parallel application traces recorded in two computing centers, namely SDSC (San Diego Supercomputer Center) and CTC (Cornell Theory Center), are used to evaluate the IBL model. The most relevant input attributes, selected according to [4], were used. The workload traces were organized through a number of disjoint sets. Each of these sets was partitioned considering $2 / 3$ as experience base and $1 / 3$ for testing, and a partial mean prediction error was computed for each set. The main IBL algorithm parameters are the neighborhood size $K$, which defines the number of relevant instances used for computing the estimate, and the $\sigma^{2}$, which defines the Gaussian function slope and the weighting values. The values experimented for $K$ were $5,10,25$ and 50. For $\sigma^{2}$, the values were $0.125,0.250,0.500,1.000$ and 2.000. The IBL algorithm achieved mean prediction errors that are between 50 and 58 percent of mean application run times (Table 2). For all workload traces, the best $K$ value founded was 5 and there was not statistical difference among the prediction errors obtained by the experimented $\sigma$ values. Nevertheless, it was observed a tendency for more higher prediction errors as the $\sigma^{2}$ value increases. The Table 3 shows the best IBL model prediction errors obtained compared to five previous approaches [12] [3] [4](the mean absolute error is used in order to compare the results with previous work).

Table 1. PSTSWM parallel application results

\begin{tabular}{|c|c|c|c|c|c|c|c|c|}
\hline \multirow[b]{2}{*}{ Attribute } & \multicolumn{4}{|c|}{$\begin{array}{l}\text { ART-2A LTM centroid traces } \\
\text { Cluster }(k)\end{array}$} & \multicolumn{4}{|c|}{ Significance Matrix } \\
\hline & 1 & 2 & 3 & 4 & 1 & 2 & 3 & 4 \\
\hline CPU time (user) & 0.003 & 0.12 & & 0.09 & $0.30 \%$ & $10.94 \%$ & $100.00 \%$ & $6.26 \%$ \\
\hline $\mathrm{I} / \mathrm{O}(\mathrm{By}$ & 0.00 & 0.00 & 0.00 & 0.00 & $0.00 \%$ & $0.00 \%$ & $0.00 \%$ & $0.00 \%$ \\
\hline I/O (Bytes write) & 0.00 & 0.00 & 0.00 & 0.00 & $0.00 \%$ & $0.00 \%$ & $0.00 \%$ & $0.00 \%$ \\
\hline Network (TCP bytes read) & 1.00 & 0.00 & 0.00 & 0.70 & $99.70 \%$ & $0.00 \%$ & $0.00 \%$ & $46.87 \%$ \\
\hline Network (TCP bytes write) & 0.00 & 0.99 & 0.00 & 0.70 & $0.00 \%$ & $89.06 \%$ & $0.00 \%$ & $46.87 \%$ \\
\hline Network (UDP bytes read) & 0.00 & 0.00 & 0.00 & 0.00 & $0.00 \%$ & $0.00 \%$ & $0.00 \%$ & $0.00 \%$ \\
\hline Network (UDP bytes write) & 0.00 & 0.00 & 0.00 & 0.00 & $0.00 \%$ & $0.00 \%$ & $0.00 \%$ & $0.00 \%$ \\
\hline Frequency & 50070 & & & 30 & & & & \\
\hline
\end{tabular}

Table 2. Experiments details

\begin{tabular}{lcccc}
\hline Workload & Experience base size & Test size & Samples & Percentage of Mean Runtime Error \\
\hline SDSC95 & 716 & 369 & 70 & $57.46 \pm 1.06$ \\
SDSC96 & 421 & 210 & 60 & $55.40 \pm 1.18$ \\
SDSC2000 & 621 & 320 & 60 & $50.05 \pm 1.59$ \\
CTC & 688 & 355 & 60 & $50.45 \pm 1.29$ \\
\hline
\end{tabular}

\section{Conclusions}

This paper presented two models for acquiring knowledge in parallel applications. The aim of these models is to improve scheduling decisions, supporting the scheduler 
Table 3. IBL algorithm results compared to previous work

\begin{tabular}{|c|c|c|c|c|c|c|}
\hline \multirow{3}{*}{ Workload } & \multicolumn{2}{|c|}{ Downey } & Gibbons & \multicolumn{2}{|c|}{ Smith } & \multirow{3}{*}{ IBL } \\
\hline & Median & Average & Fixed & Greedy & Genetic & \\
\hline & Lifetime & Lifetime & Templates & Search & Search & \\
\hline SDSC95 & 82.44 & 171.00 & 74.05 & 67.63 & 59.65 & 33.98 \\
\hline SDSC96 & 102.04 & 168.24 & 122.55 & 76.20 & 74.56 & 58.32 \\
\hline CTC & 179.46 & 201.34 & 124.06 & 118.05 & 106.73 & 109.06 \\
\hline
\end{tabular}

with knowledge about the resource usage patterns of parallel applications. Through the experiments, the local knowledge acquisition model presented a good classification performance. Another aspect observed is the robustness of this model at the different computational loads and processing elements configurations [5]. The global knowledge acquisition model presented a great potential to define similarity among parallel applications, weighting the more relevant instances in an experience base to generate an output attribute estimate. Our IBL approach achieved a good prediction performance when compared to both static and adaptive templates prediction approaches.

Acknowledgments. This project is supported by CAPES/PICDT program. The authors would like to thank Reagan Moore, Allen Downey, Victor Hazelwood (San Diego Supercomputer Center) and the Cornell Theory Center for graciously providing the workload traces used in this work. Particular thanks to Warren Smith, for providing his prediction software.

\section{References}

1. Silva, F.A.B.D., Scherson, I.D.: Improving Parallel Job Scheduling Using Runtime Measurements. In Feitelson, D.G., Rudolph, L., eds.: Job Scheduling Strategies for Parallel Processing. Springer Verlag (2000) 18-38 Lect. Notes Comput. Sci. vol. 1911.

2. Naik, V.K., Setia, S.K., Squillante, M.S.: Processor Allocation in Multiprogrammed Distributed-memory Parallel Computer Systems. Journal of Parallel and Distributed Computing 47 (1997) 28-47

3. Gibbons, R.: A Historical Application Profiler for Use by Parallel Schedulers. In: Job Scheduling Strategies for Parallel Processing. Springer Verlag (1997) 58-77

4. Smith, W., Foster, I.T., Taylor, V.E.: Predicting Application Run Times Using Historical Information. In: JSSPP. (1998) 122-142

5. Senger, L.J., Santana, M.J., Santana, R.H.C.: A new approach fo acquiring knowledge of resource usage in parallel applications. In: Proceedings of International Symposium on Performance Evaluation of Computer and Telecommunication Systems (SPECTS'2003). (2003) 607-614

6. Carpenter, G.A., Grossberg, S., Rosen, D.B.: ART 2-A: An Adaptive Resonance Algorithm for Rapid Category Learning and Recognition. Neural Networks 4 (1991) 494-504

7. Whiteley, J.R., Davis, J.F.: Observations and problems applying ART2 for dynamic sensor pattern interpretation. IEEE Transactions on Systems, Man and Cybernetics-Part A: Systems and Humans 26 (1996) 423-437

8. Ultsch, A.: Self-organising neural networks for monitoring and knowledge acquisition of a chemical process. In: Proceedings of ICANN-93. (1993) 864-867 
9. Foster, I.T., Worley, P.H.: Parallel algorithms for the spectral transform method. SIAM J. Sci. Stat. Comput. 3 (1997) 806-837

10. Aha, D.W., Kibler, D., Albert, M.K.: Instance-based learning algorithms. Machine Learning (1991) 37-66

11. Feitelson, D.G., Rudolph, L., Schwiegelshohn, U., Sevcik, K.C., Wong, P.: Theory and Practice in Parallel Job Scheduling. In: Job Scheduling Strategies for Parallel Processing. Volume 1291. Springer Verlag (1997) 1-34 Lect. Notes Comput. Sci. vol. 1291.

12. Downey, A.: Predicting queue times on space-sharing parallel computers. In: Proceedings of International Parallel Processing Symposium. (1997) 